\title{
Commissioning and prospects of the first GEM station at the CMS experiment
}

\author{
Giovanni Mocellin \\ a Physics Institute III A, RWTH Aachen University, \\ Sommerfeldstrasse 16, Aachen, Germany
}

E-mail: giovanni.mocellin@physik.rwth-aachen. de

The CMS Collaboration has been developing a Gas Electron Multiplier (GEM) detector in the endcap regions of the CMS muon system to maintain the high level of performance achieved during Run 2 in the challenging environment of the high-luminosity phase of the LHC (HL-LHC). The GEM detectors at endcap station 1 (GE1/1) were installed during the second long shutdown. The technical and operational challenges of large-area GEM detectors have been identified during the commissioning of five GEM super chambers ("slice test") in Run 2. This led to a modification in its system design. A test with cosmic-ray muons is the final stage of quality control before the full-scale installation into the CMS detector. We review the performance of muon detection in the slice test, an improvement of the readout system, commissioning status, and prospects for the muon trigger for Run 3.

40th International Conference on High Energy physics - ICHEP2020

July 28 - August 6, 2020

Prague, Czech Republic (virtual meeting)

\footnotetext{
$\ddagger$ On behalf of the CMS Collaboration

*Speaker
} 


\section{Introduction}

The Compact Muon Solenoid (CMS) is a general purpose particle physics detector at the CERN Large Hadron Collider (LHC) [1]. After an upgrade of the LHC that will take place during the Long Shutdown 3, from 2027 a new phase of collision data taking will start. In the HL-LHC runs, the LHC will deliver an instantaneous luminosity up to $5 \times 10^{34} \mathrm{~cm}^{-2} \mathrm{~s}^{-1}$ to its experiments. This will require an upgrade of the detectors, especially in forward regions, to be able to cope with a higher number of collisions per bunch crossing, larger trigger rates, and greater absorbed radiation dose. In the CMS endcap regions dedicated to the muon detection, the present Cathode Strip Chambers (CSCs) and Resistive Plate Chambers (RPCs) will be complemented by new generations of detectors: Gas Electron Multiplier (GEM) detectors will be deployed in the first two stations, while the remaining two will be equipped with improved Resistive Plate Chambers (iRPCs). As shown in Fig. 1a, they will cover the region $1.5<|\eta|<2.8$ [2, 3], ensuring increased redundancy, improved $p_{T}$ measurements and control of the increased trigger rate, see Fig. $1 \mathrm{~b}$.

These proceedings will have their focus on the GEM upgrade, particularly on the recently installed GE1/1 detectors.

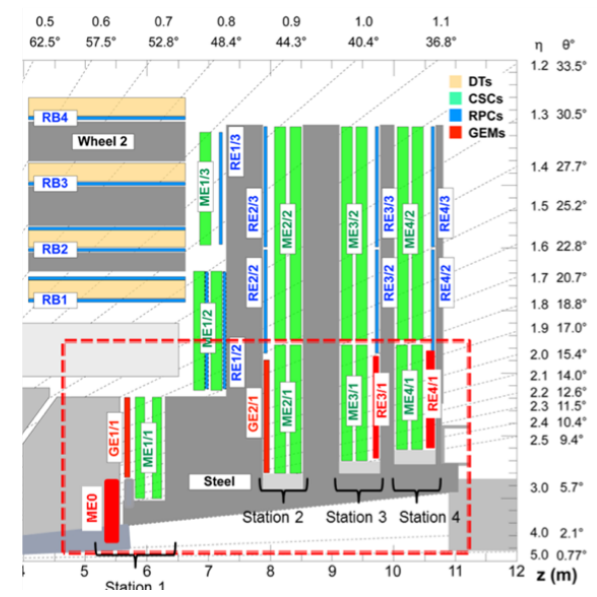

(a) Overview in the transverse plane of the CMS muon endcap upgrade.

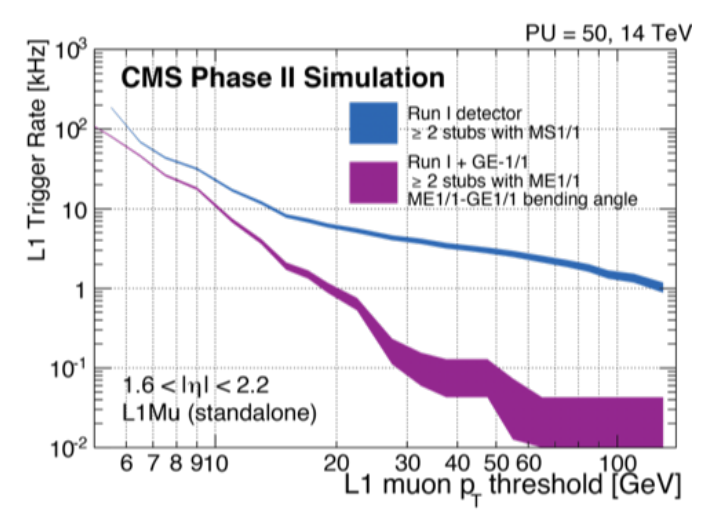

(b) Muon trigger rate reduction with the introduction of GE1/1.

Figure 1: CMS muon endcap upgrade.

\section{GEM technology}

GEMs are a gaseous detector technology invented by F. Sauli in 1997 [4]. Their key element is the GEM foil, consisting of a $50 \mu \mathrm{m}$ thick copper-clad polyimide foil. Holes are etched in a hexagonal pattern with a diameter of $70 \mu \mathrm{m}$ and a pitch of $140 \mu \mathrm{m}$, as in Fig. 2a. A CMS GEM detector is composed of a drift board, a readout PCB, and a stack of three GEM foils, named 'triple-GEM'. Incident ionizing particles generate electron-ion pairs in the gas, and thanks to the applied high voltage, the electrons are collected from the drift gap and guided towards the foils. Inside the GEM holes, an electric field of around $60 \mathrm{kV} / \mathrm{cm}$ causes avalanches to form and the electrons are multiplied. The three stages of amplification ensure a final gas gain on the order of $10^{4}$. 
The detector characteristics that make the GEM technology suitable for the CMS muon endcaps are the rate capability of the order of $M H z / \mathrm{cm}^{2}$ and the efficiency for minimum ionizing particles (MIPs) greater than 97\%. In addition, the radiation hardness was proven up to a level of operation with an integrated luminosity of the LHC of higher than $3000 \mathrm{fb}^{-1}$ [6].

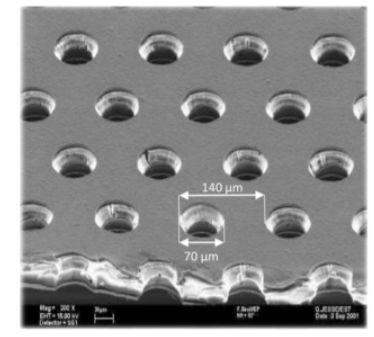

(a) SEM microscope picture of a GEM foil.

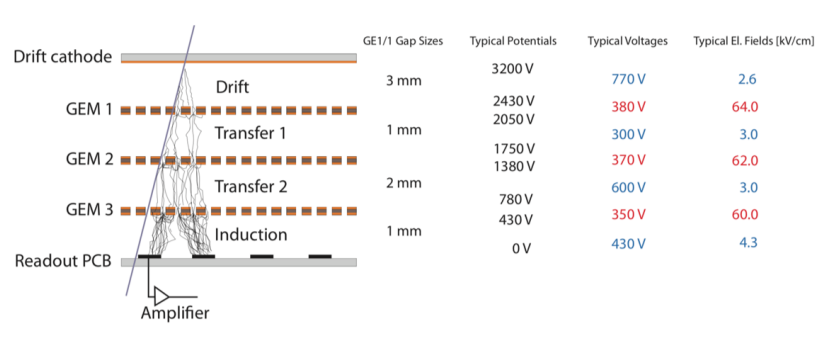

(b) Schematic view of a GEM chamber, with typical voltages applied to the electrodes (CMS GEM).

Figure 2: GEM detectors.

\section{GE1/1 chamber}

The GE1/1 detectors are designed to fit within the the first disk and first ring of the CMS endcap, covering $1.5<|\eta|<2.2$, as presented in Fig. 3a. The gas mixture employed is $\mathrm{Ar} / \mathrm{CO}_{2}$ at a $70 \% / 30 \%$ ratio. Their trapezoidal active area of about $0.5 \mathrm{~m}^{2}$ covers a much larger surface than the previously produced GEMs. This has copious implications on their operation.

The signals produced by the traversing particles are induced on radial strips, amounting to a total of 3072 per chamber. Groups of 128 strips are readout by 24 analog to digital frontend chips (VFATs [5]), organized into 8 partitions along the $\eta$ direction and 3 along $\phi$, see Fig. $3 b$.

In total, 144 single chambers are coupled into 72 super chambers. Each covers $10.15^{\circ}$ in the disk, with neighboring chambers designed to overlap. Short and long chambers alternate around the disks to cope with the mechanical constraints of the CMS endcap, as in Fig. 3c.

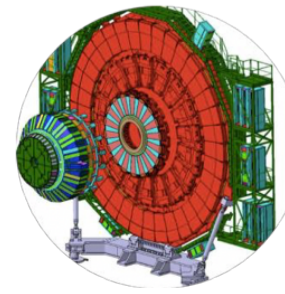

(a) GE1/1 ring in the CMS endcaps.

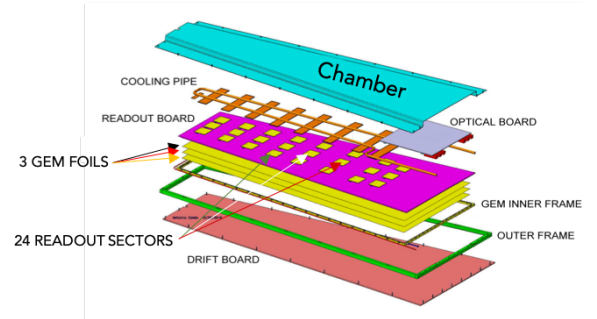

(b) GE1/1 chamber.

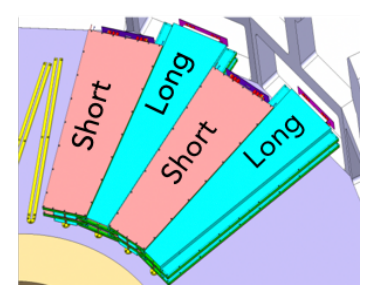

(c) Super chambers arrangement.

Figure 3: Construction and placement of GE1/1.

\section{Slice test}

The first implementation of the GE1/1 chambers in CMS took place in 2017 - 2018, called the 'GEM slice test'. Five detectors were installed in one of the two endcaps, arranged as in Fig. 4a. 
The main goals of the demonstrator were: the acquisition of installation and operation experience, as well as the development of the integration with central CMS online systems (Detector Control System, Data Acquisition and Data Quality Monitoring).

The major targets were met and one of the fundamental results is shown in Fig. 4b. The detection efficiency for the eigth $\eta$ partitions is presented for the chamber installed in Layer 2 of Slot 28, which was computed by analyzing the Run 22018 data, extrapolating global muons tracks with $p_{T}>20 \mathrm{GeV}$ to the GE1/1 station. The experience gained during the operations was also

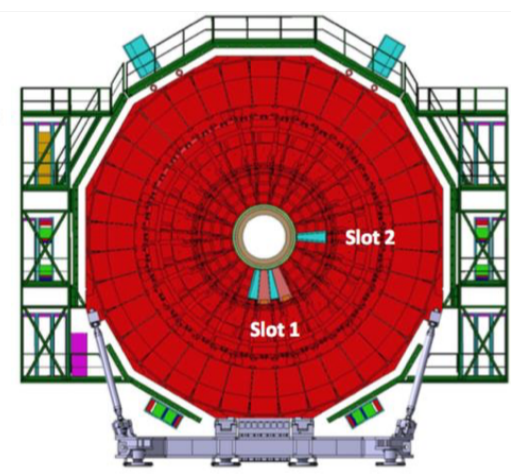

(a) Slice test detector slots.

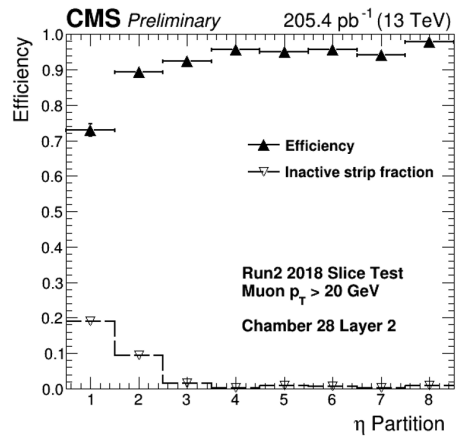

(b) Detection efficiency per $\eta$ partition for the chamber 28 layer 2.

Figure 4: GEM Slice Test.

essential to uncover issues present on the demonstrating detectors and allow the chance to improve before the final installation. The most significant finding was the high sensitivity of the on-chamber electronics to the high voltage discharges happening in the detector that propagated to the readout strips. When a spark had the chance to deliver its energy to the VFAT2 channels' inputs, they could be damaged permanently and create a dead area.

The number of lost channels during the entire period of slice test operations is shown in Fig. 5a. Large variations across the detectors can be seen, and it mostly depended on the stability of the high voltage of each of the chambers. This initiated a barrage of studies aimed minimizing the probability of discharges damaging channels. The new version of the frontend chip (VFAT3), was tested equipping it with many possible solutions that included additional resistors or diodes as channel input protection. Considering the results in Fig. 5b, the final version of the VFAT3 (HV3b_V3) was designed having a $470 \Omega$ input protection resistor for each of the 128 channels. The offered mitigation reduced the chance of having a propagating discharge damaging the VFAT channels from about $90 \%$ down to $3 \%$.

\section{Production and quality control of the GE1/1 detectors}

The journey of a GE1/1 detector starts with the assembly of the single chambers in seven countries around the world (CERN, Belgium, Germany, India, Italy, Pakistan, USA). Following production, a series of five quality control tests are performed to ensure their gas tightness, HV stability and assess gas gain performance. The chambers are finally shipped to CERN, where they are equipped with the final optimized frontend readout electronics, which gets tested during the 7th 

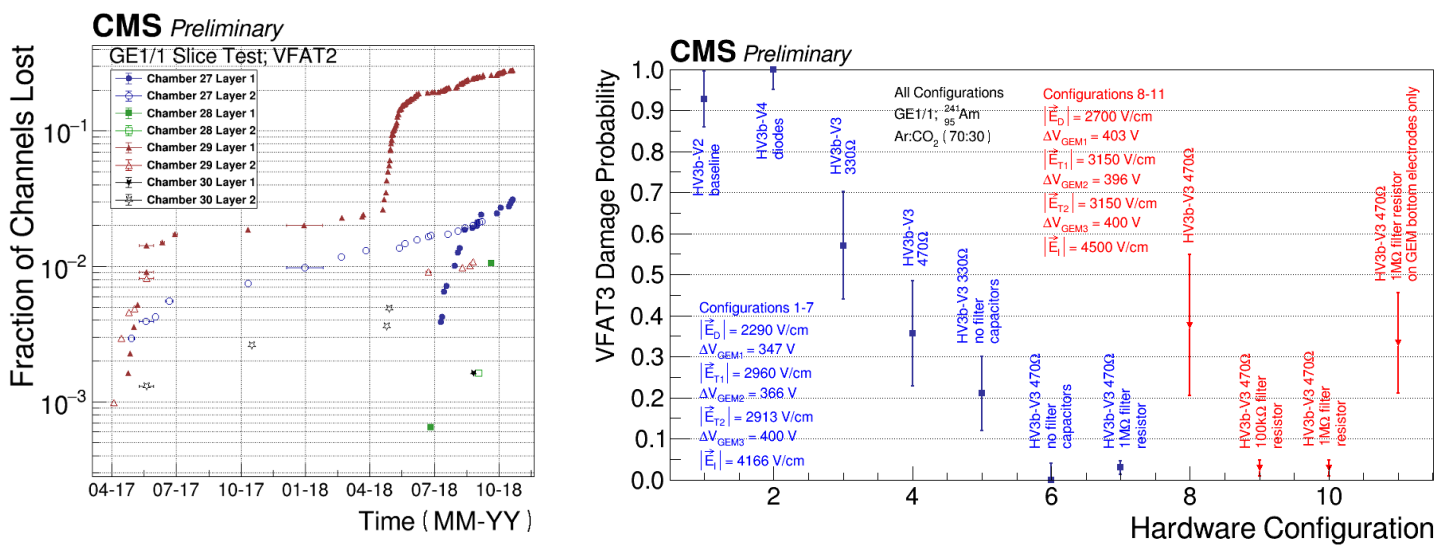

(a) Fraction of channels lost per chamber dur- (b) Mitigation studies to minimize the VFAT channel loss due to propaing slice test operations. gating discharges.

Figure 5: Slice test issues and consequent studies.

QC step. Super chambers are then built by pairing single chambers matched to gas gain. The last and final major quality control test (QC8) assesses their performance in terms of efficiency using a dedicated cosmic rays stand at CERN.

The structure of the stand, in Fig. 6a, foresees 15 slots for the super chambers and two layers of plastic scintillators on top and bottom, used as triggers. The services provided to the detectors are as the ones in CMS. After a full configuration of the on-chamber electronics and a high voltage training, cosmic muon runs are taken scanning the HV working point. The data is then analyzed reconstructing the muon tracks, as in Fig. 6b, and extracting their performance parameters.

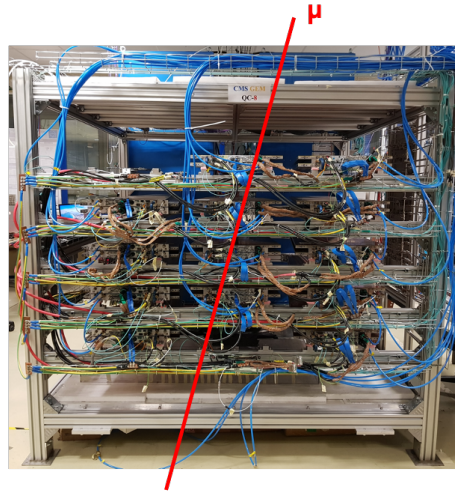

(a) Cosmic stand structure.

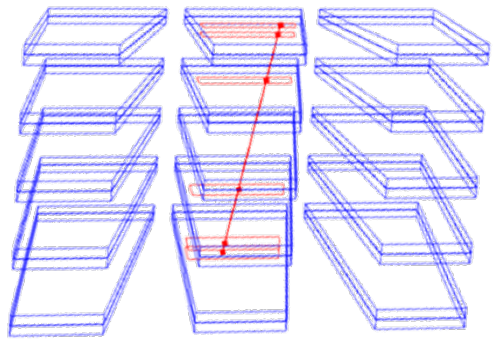

(b) Event display: reconstructed cosmic muon track.

Figure 6: GEM cosmic ray stand test.

Examples of typical results can be seen in Figs. 7a, 7b and 7c. High voltage scans are performed and efficiency and spatial resolution are calculated. The plateau of efficiency at $97 \%$ starts at around $680 \mu \mathrm{A}$ equivalent divider current, to which corresponds a spatial resolution of about $160 \mu \mathrm{rad}$. These values are well within the requirements [2] and ensure the ability to provide a substantial improvement to the CMS muon endcap trigger. 


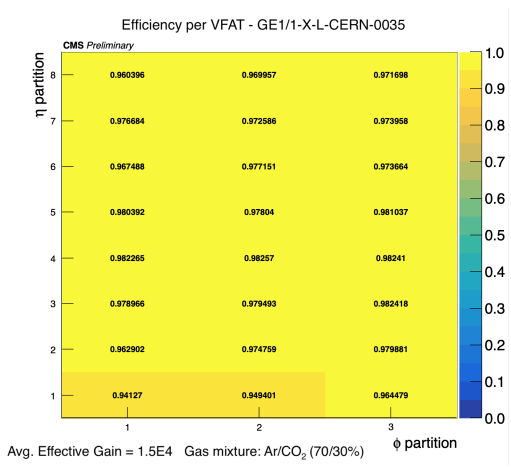

(a) Efficiency per VFAT.

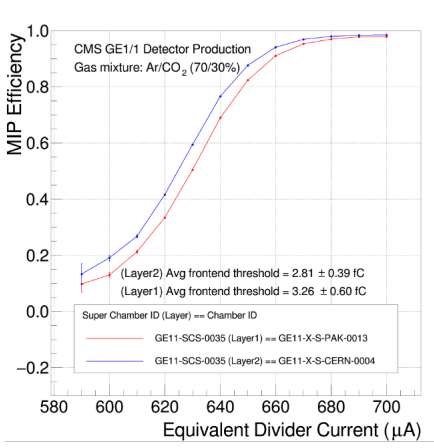

(b) Efficiency vs HV.

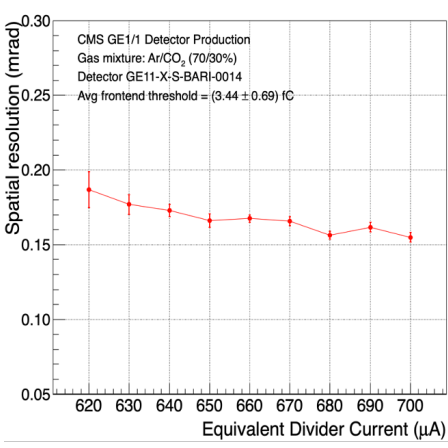

(c) Spatial resolution vs HV.

Figure 7: GE1/1 performance studied during the QC8.

\section{Installation and commissioning of the GE1/1 detectors}

From July to September in 2019, all 36 GE1/1 detectors were installed in the negative endcap of CMS. Subsequently, the commissioning phase started. All the frontend channels were checked with test pulses to ensure proper communication, and the noise level was assessed. The detector installation in the positive endcap of the CMS was successfully completed in August-September 2020. The plans for the commissioning foresee a complete integration of DCS, DAQ, DQM in the central CMS system, the configuration of the frontend and backend electronics, the high voltage training of the chambers, and latency and efficiency scans to determine optimal working point.

\section{Conclusions}

The GEM technology and the GE1/1 design have been described, together with the production and the quality control tests. Issues found during the slice test have been presented, as well as the mitigation studies and the implemented solution. The most significant results from the cosmic ray stand test have been shown, demonstrating excellent performance of the GE1/1 detectors. The installation of all the chambers in the CMS experiment was successfully completed and the commissioning phase has started.

\section{References}

[1] CMS Collaboration, The CMS experiment at the CERN LHC, JINST 3 S08004, 2008.

[2] A. Colaleo et al., CMS Technical Design Report for the Muon Endcap GEM Upgrade, CERNLHCC-2015-012; CMS-TDR-013, 2015.

[3] CMS collaboration, The Phase-2 Upgrade of the CMS Muon Detectors, CERN-LHCC-2017012 ; CMS-TDR-016, 2017.

[4] F. Sauli, The gas electron multiplier (GEM): Operating principles and applications, Nuclear Instruments and Methods in Physics Research A 805, Pages 2-24, 2016.

[5] P. Aspell et al., VFAT3: A Trigger and Tracking Front-end ASIC for the Binary Readout of Gaseous and Silicon Sensors, IEEE Nuclear Science Symposium and Medical Imaging Conference Proceedings (NSS/MIC), 2018.

[6] F. Fallavollita, Triple-Gas Electron Multiplier technology for future upgrades of the CMS experiment: construction and certification of the CMS GE1/1 detector and longevity studies, CERN-THESIS-2018-349, 2018. 